\title{
Phase space gaps and ergodicity breaking in systems with long range interactions
}

\author{
Freddy Bouchet ${ }^{1,2}$, Thierry Dauxois ${ }^{1}$, David Mukamel ${ }^{1,3}$, Stefano Ruffo ${ }^{1,4}$ \\ 1. Université de Lyon, CNRS, École Normale Supérieure de Lyon \\ Laboratoire de Physique, 46 Allée d'Italie, 69364 Lyon cedex 07, France \\ 2. Institut Non Linéaire de Nice, CNRS, 1361 route des Lucioles 06560 Valbonne, France. \\ 3. Department of Physics of Complex Systems, The Weizmann Institute of Science, Rehovot, Israel \\ 4. Dipartimento di Energetica "S. Stecco" and CSDC, \\ Università di Firenze and INFN, Via S. Marta, 3 I-50139, Firenze, Italy
}

(Dated: November 12, 2018)

\begin{abstract}
We study a generalized isotropic XY-model which includes both two-spin and four-spin meanfield interactions. This model can be solved in the microcanonical ensemble. It is shown that in certain parameter regions the model exhibits gaps in the magnetization at fixed energy, resulting in ergodicity breaking. This phenomenon has previously been reported in anisotropic and discrete spin models. The entropy of the model is calculated and the microcanonical phase diagram is derived, showing the existence of first order phase transitions from the ferromagnetic to a paramagnetic disordered phase. It is found that ergodicity breaking takes place both in the ferromagnetic and the paramagnetic phases. As a consequence, the system can exhibit a stable ferromagnetic phase within the paramagnetic region, and conversely a disordered phase within the magnetically ordered region.
\end{abstract}

PACS numbers: 05.70.Fh Phase transitions: general studies

\section{INTRODUCTION}

The aim of this paper is to shed light on a dynamical feature which distinguishes systems with long range interactions from those with short range ones. It is well known that the attainable region in the space of extensive thermodynamic parameters is always convex when only short range interactions are present. Consider, for example, two magnetic subsystems with the same potential energy $V$ but with two different magnetization values $m_{1}$ and $m_{2}$ (notice that, instead of the magnetization, we might have chosen any other order parameter). Introducing a parameter $\lambda$ which depends on the relative size of the subsystems, it is possible to get any intermediate value of the magnetization $m$ by just combining the two subsystems with appropriate weights, such that $m=\lambda m_{1}+(1-\lambda) m_{2}$, while the potential energy is kept constant to $V$. It is important to stress that this convexity property is satisfied only if the system is additive, since the interaction energy between the two subsystems has been neglected. This can safely be done only for short range interacting systems in the large volume limit. Moreover, convexity implies that the accessible region in the space of thermodynamic parameters is connected.

In contrast, systems with long range interactions [1] are not additive. As a consequence, the convexity property may be violated, and then also connectivity. This feature has profound consequences on the dynamics. Gaps may open up in the order parameters space. Such gaps have been recently reported in a class of anisotropic $X Y$ models [2, 3] and for a discrete spin system [4]. Since the accessible region in the order parameters space is no more connected, ergodicity breaking naturally appears when a continuous microcanonical dynamics is considered. This is the property we discuss in this paper. More specif- ically, the question we would like to address is that of locating the region in the phase diagram of the model where we expect ergodicity breaking to occur. We will argue that ergodicity breaking occurs in regions of parameters where metastable states exist. In such regions, one may also generically expect first order phase transitions. Thus, we conclude that the breakdown of order parameters space connectivity is generically associated with first order phase transitions. Moreover, we conjecture that the intersection between first order phase transition lines and the boundary of the parameter region where the order parameters space is disconnected, occurs at non smooth (singular) points of the boundary itself. We also conjecture that the same is true for the metastability line.

In recent years, models describing classical rotators with all-to-all mean-field interaction have been intensively studied in order to explain general dynamical features of long-range interacting systems 1]. The most celebrated of such models is the so-called Hamiltonian Mean Field (HMF) model, where rotators denoted by the angle $\theta_{i}, i=1, \ldots, N$ are coupled through a long-range mean-field potential

$$
W=-\frac{J}{2 N} \sum_{i, j} \cos \left(\theta_{i}-\theta_{j}\right) .
$$

The interaction is ferromagnetic for positive $J$-values, and antiferromagnetic otherwise. Defining the complex order parameter (with $i$ the imaginary unit)

$$
\vec{m}=\left(m_{x}+i m_{y}\right)=\frac{1}{N} \sum_{n} \exp \left(i \theta_{n}\right),
$$

the potential per particle $V=W / N$ can be rewritten as $-J m^{2} / 2$ where $m=|\vec{m}|$ is the modulus of the magnetization. The total energy is finally obtained by adding a 
kinetic energy term to the above potential so that we get the Mean-Field Hamiltonian

$$
H=\sum_{n=1}^{N} \frac{p_{n}^{2}}{2}+N V .
$$

In this formulation, the model can also be thought of as describing a system of particles with unit mass, interacting through the mean-field coupling (the names rotator and particle might equivalently be used). Note that a system with non-unit mass may be reduced to the above system by rescaling time. As the parameter $J$ might be removed by a suitable energy rescaling, we will omit it in the remainder of the paper. Within the Hamiltonian framework, both the energy density $E=H / N$ and the total momentum $P=\sum_{n} p_{n}$ are conserved quantities and they are fixed by the initial conditions.

In this paper, we study a generalized mean-field Hamiltonian [5] with potential

$$
V(m)=\frac{m^{2}}{2}-K \frac{m^{4}}{4},
$$

which reduces to the antiferromagnetic HMF model $(J=$ -1 ) in the particular case $K=0$. In addition to the first antiferromagnetic term, we consider a ferromagnetic fourth order term in the magnetization, whose intensity is controlled by a positive constant $K$. In the regime of large values of the parameter $K$, a magnetized state would be favorable because of the ferromagnetic coupling while, for small values of $K$, the antiferromagnetic coupling dominates and leads to a non-magnetic state. As we shall demonstrate below, there exists a range of the parameter $K$ for which the model exhibits a first order phase transition between a paramagnetic phase at high energies and a ferromagnetic phase at low energies. In both phases there are regions in the $(E, K)$ plane in which the accessible magnetization interval exhibits a gap, resulting in breaking of ergodicity.

The organization of the paper is as follows. In Section III, we first address the issue of the accessible magnetization interval in this model. In the following Section III we present the statistical mechanics of the model. In particular, we study the phase diagram and the conditions for phase transitions to occur. In Section IV] we study the dynamical consequences of this ergodicity breaking. Finally, Section $\nabla \nabla$ will be devoted to conclusions and perspectives.

\section{PHASE SPACE CONNECTIVITY}

Let us study the phase space structure and the phase diagram of this model. The specific kinetic energy $E_{K}=$ $E-V(m)$ is by definition a non negative quantity, which implies that

$$
E \geq V(m)=m^{2} / 2-K m^{4} / 4 .
$$

We will show that as a result of this condition not all the values of the magnetization $m$ are attainable in a certain region in the $(E, K)$ plane; a disconnected magnetization domain might be indeed a typical case. As explained below, this situation is the one of interest. Let us characterize the accessible domains in the $(E, K)$ plane more precisely by analyzing the different regions.

For $K<1$, the local maximum of the potential energy $V$ is not located inside the magnetization interval $[0,1]$ (see Fig. 1a). The potential being a strictly increasing function of the magnetization, the maximum is reached at the extremum $m=1$. The complete interval $[0,1]$ is thus accessible, provided the energy $E$ is larger than $V(1)$ : the corresponding domain is in $R 1$ defined in Fig. 2, The horizontally shaded region is forbidden, since the energy is lower than the minimum of the potential energy $V(0)=0$. Finally, the intermediate region $0<E<V(1)$ in included in the region $R$ 2: it is important to emphasize that only the interval $\left[0, m_{-}(E, K)\right]$, where $m_{ \pm}(E, K)=[(1 \pm \sqrt{1-4 E K}) / K]^{1 / 2}$, is accessible. Larger magnetization values correspond to a potential energy $V(m)$ larger than the total energy $E$, which is impossible. Figure 1 also displays the value $m_{-}$corresponding to an energy in the intermediate region $R$ 2.

For $K \geq 1$, the specific potential energy $V$ has a maximum $V_{\max }=1 / 4 K$ which is reached at $0<m_{\max }=$ $1 / \sqrt{K} \leq 1$. Figures $1 \mathrm{~b}$ and $1 ;$, where the potential energy (44) is plotted $v s$ the magnetization $m$, present such cases. For an energy $E$ larger than the critical value $V_{\max }$, condition (5) is satisfied for any value of the magnetization $m$. The complete interval $[0,1]$ is thus accessible for the magnetization $m$. This region is $R 1$ represented in Fig. 2,

Let us now consider the cases for which $E \leq V_{\max }$. As discussed above, the minimum $V_{\min }$ of the potential energy is also important to distinguish between the different regions. For $1 \leq K \leq 2$ (see Fig. 1b), the minimum of $V(m)$ corresponds to the non-magnetic phase $m=0$ where $V(0)=0$. The quadrilled region shown in Fig. 2 , which corresponds to negative energy values, is thus nonaccessible. On the contrary, positive energy values are possible and correspond to very interesting cases, since only sub-intervals of the complete magnetization interval $[0,1]$ are accessible. There are however two different cases:

- for $0<V(1)<E<V_{\max }$, the domain of possible magnetization is $\left[0, m_{-}(E, K)\right] \cup\left[m_{+}(E, K), 1\right]$. The above conditions are satisfied in $R 3$ of Fig. 2

- for $0<E<V(1)$, only the interval $\left[0, m_{-}(E, K)\right]$ satisfies condition (5). This takes place within $R 2$ of Fig. 2.

For the domain $2 \leq K$, the minimum of the potential energy is attained at the extremum, $m=1$, implying $E>V(1)=1 / 2-K / 4$. The vertically shaded region is thus forbidden. In the accessible region two cases can be identified: 
a)

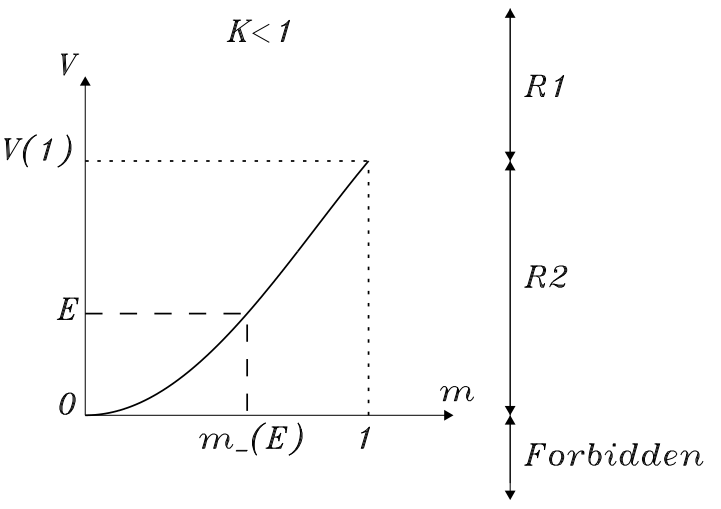

b)

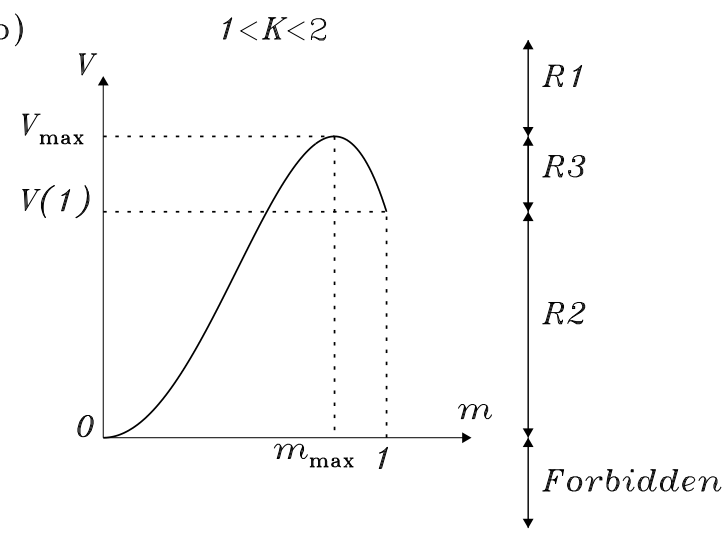

c)

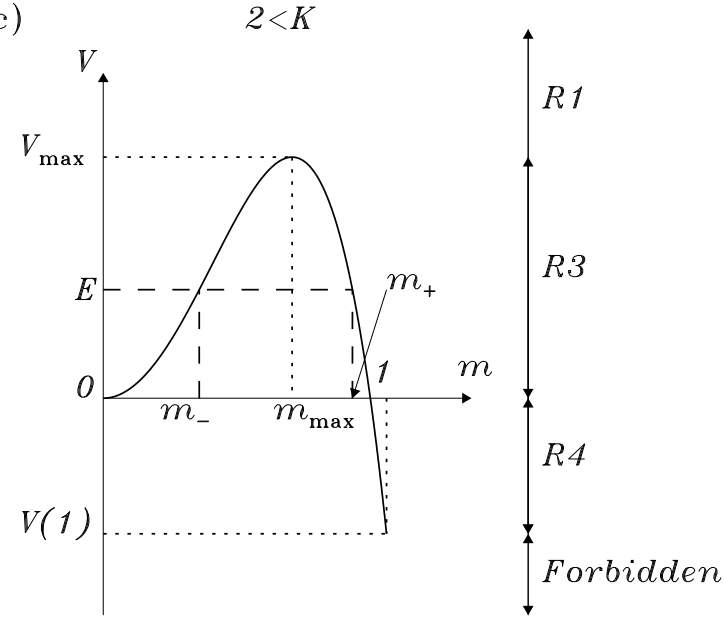

FIG. 1: Specific potential energy $V$ vs. magnetization $m$ for three different cases: $K<1$ (panel a), $1<K<2$ (panel b) and $2<K$ (panel c). The location of the maximal magnetization $m_{\max }$ and the corresponding potential energy $V_{\max }$ are shown (see text). In panels (a) and (c), two examples of the location of the critical magnetization $m_{ \pm}(E, K)$ is indicated for energy values $E$ in the intermediate regions.

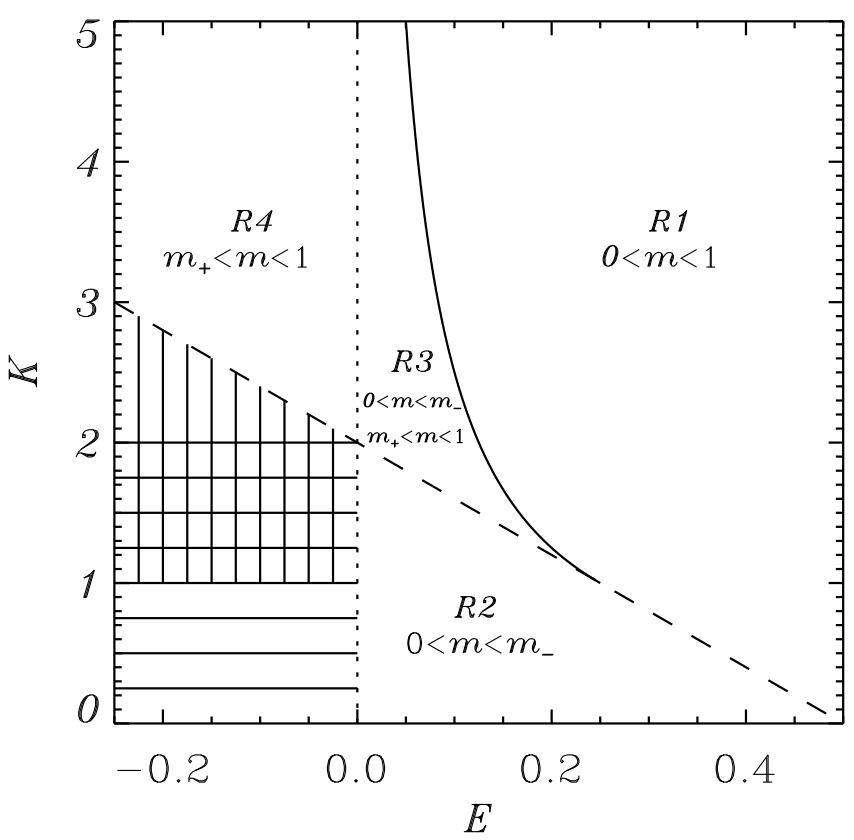

FIG. 2: The $(E, K)$ plane is divided in several regions. The solid curve corresponds to $K=1 /(4 E)$, the oblique dashed line to $K=2-4 E$, while the dotted one to $K=1$. The vertically shaded, quadrilled, and horizontally shaded regions are forbidden. The accessible magnetization interval in each of the four regions is indicated (see text for details).

- for $V(1)<E<0$, only the interval $\left[m_{+}(E, K), 1\right]$ satisfies condition (5). It is important to note that $m_{+}(E, K) \leq 1$ provided $E \geq 1 / 2-K / 4$. These cases correspond to $R 4$.

- for $0 \leq E \leq V_{\max }$, the two intervals $\left[0, m_{-}(E, K)\right]$ and $\left[m_{+}(E, K), 1\right]$ satisfy condition (5), corresponding to R3 of Fig. 2

In summary, the complete magnetization interval $[0,1]$ is accessible only in the region $R 1$. In $R 2$, only $\left[0, m_{-}\right]$ is accessible, while only $\left[m_{+}, 1\right]$ is accessible in $R 4$. Finally, we note that the phase space of the system is not connected in the region $R 3$. Indeed, the magnetization cannot vary continuously from the first interval $\left[0, m_{-}\right]$ to the second one $\left[m_{+}, 1\right]$, although both are accessible. These restrictions yield the accessible magnetization domain shown in Fig. 3. The fact that for a given energy the phase space is disconnected implies ergodicity breaking for the Hamiltonian dynamics. It is important to emphasize that the discussion above is independent of the number of particles and ergodicity is expected to be broken even for a finite (but sufficiently large) $N$. 


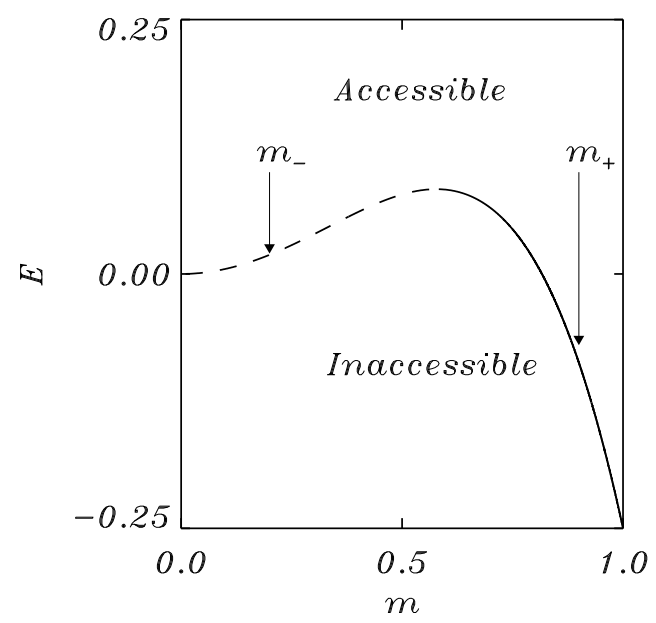

FIG. 3: Accessible region in the $(m, E)$ plane for $K=3$. For energies in a certain range, a gap in the accessible magnetization values is present and defined by the two boundaries $m_{ \pm}(E, K)$.

\section{STATISTICAL MECHANICS}

We have thus found that in certain regions in the $(E, K)$ plane, the magnetization cannot assume any value in the interval $[-1,1]$. For a given energy there exists a gap in this interval to which no microscopic configuration can be associated. In this Section, we study the statistical mechanics of model (4), by considering the microcanonical measure

$$
\mu(E, N)=\prod_{k=1}^{N} \mathrm{~d} \theta_{k} \mathrm{~d} p_{k} \delta(H-N E) .
$$

The probability $P(m)$ to observe the magnetization $m$ can be obtained for large values of the number $N$ of particles using large deviation techniques, in a similar way as it was derived for the HMF model [6]. Here again, the appropriate global variables are the magnetization $m$ and the mean kinetic energy $E_{K}=\sum_{n} p_{n}^{2} / N$. Cramér's theorem allows one to derive the probability distribution $P(m)$ in the large $N$-limit. The entropy per particle, for given energy and magnetization, is obtained as

$$
s(E, m)=\lim _{N \rightarrow \infty} \frac{1}{N} \ln P(m),
$$

Following the steps described in Ref. [6], one finds that the specific entropy $s$ can be expressed as

$$
s(E, m)=s_{K}(E, m)+s_{\text {conf }}(m),
$$

i.e. the sum of the momentum entropy, which is related to the kinetic energy

$$
s_{K}(E, m)=\ln \left(E_{c}\right) / 2=\frac{1}{2} \ln \left(E-\frac{m^{2}}{2}+K \frac{m^{4}}{4}\right),
$$

and of the configuration entropy which, as for the HMF model, is

$$
s_{\text {conf }}(m)=-m \phi(m)+\ln \left(I_{0}(\phi(m))\right) .
$$

Here $\phi$ is the inverse of the function $I_{1} / I_{0}$ where $I_{n}$ is the modified Bessel function of order $n$. The microcanonical thermodynamics is finally recovered solving the variational problem

$$
S(E)=\sup _{m}[s(E, m)] .
$$

First, by comparing the low and high energy regimes, it is possible to show that a phase transition is present between the two regimes. In the domain $R 4$ of Fig. 2 for very low energy $E$ (close to the limiting value $1 / 2-K / 4$ ), the accessible range for $m$ is a small interval located close to $m=1$ (see Fig. 1 ). The maximum of the entropy $s$ corresponds therefore to a magnetized state, $m_{e q}$, located very close to $m=1$ (see the top-left inset in Fig. 44). On the contrary, in the very large energy domain, the variations of entropy with respect to $m$ are dominated by the variations of the configurational entropy since the kinetic entropy (see Eq. (9) with $m$ of order one and a very large energy $E$ ) is roughly a constant around $s(E, 0)=(\ln E) / 2$. As expected, the configurational entropy is a decreasing function of the magnetization: the number of microstates corresponding to a non-magnetic macrostate being much larger that the same number for a magnetized state. The configuration entropy has therefore a single maximum located at $m_{e q}=0$. A phase transition takes place between the non-magnetic state at large energy, and a magnetic state at small energy. Moreover, as the non-magnetic state is possible only for positive energies $E$ (see Figs. 10, the transition line is located in the domain $E \geq 0$. In this region, the quantity $\partial_{m}^{2} s(E, 0)=-(1+1 /(2 E))$ is negative, which ensures that, for any value of $E$ and $K$, the non-magnetic state $m=0$ is a local entropy maximum. The latter argument allows to exclude a second order phase transition at a positive critical energy, since the second derivative $\partial_{m}^{2} s(E, 0)$ would have to vanish, which is impossible. The above argument leads to the conclusion that the phase transition must be first order.

Let us now focus on the behavior of the entropy in the region $R 3$, where the accessible range for $m$ is the union of two disconnected intervals $\left[0, m_{-}\right] \cup\left[m_{+}, 1\right]$. As discussed above, the total entropy $s(E, m)$ has a local maximum in the first interval $\left[0, m_{-}\right]$located at $m=0$ and associated with the entropy $s_{\max }^{1}=s(E, 0)=\log (E) / 2$. In the second interval $\left[m_{+}, 1\right]$, a maximum is also present with $s_{\max }^{2}=s\left(E, m_{e q}\right)$ where $m_{e q} \geq m_{+}>0$. As $s_{\max }^{1}(E)$ diverges to $-\infty$ when $E$ tends to 0 , a magnetized state is expected on the line $E=0$, as long as $s_{\text {max }}^{2}$ remains finite. Since $K=2$ is the only value for which $s_{\max }^{2}(0, K)$ diverges, the first order transition line originates at the point $B(0,2)$ in Fig. 4. Although it is possible to study analytically the asymptotic behavior of the transition line near this point, we can rather easily compute numerically 
the location of the first order transition line, represented by the dash-dotted line in Fig. 4 .

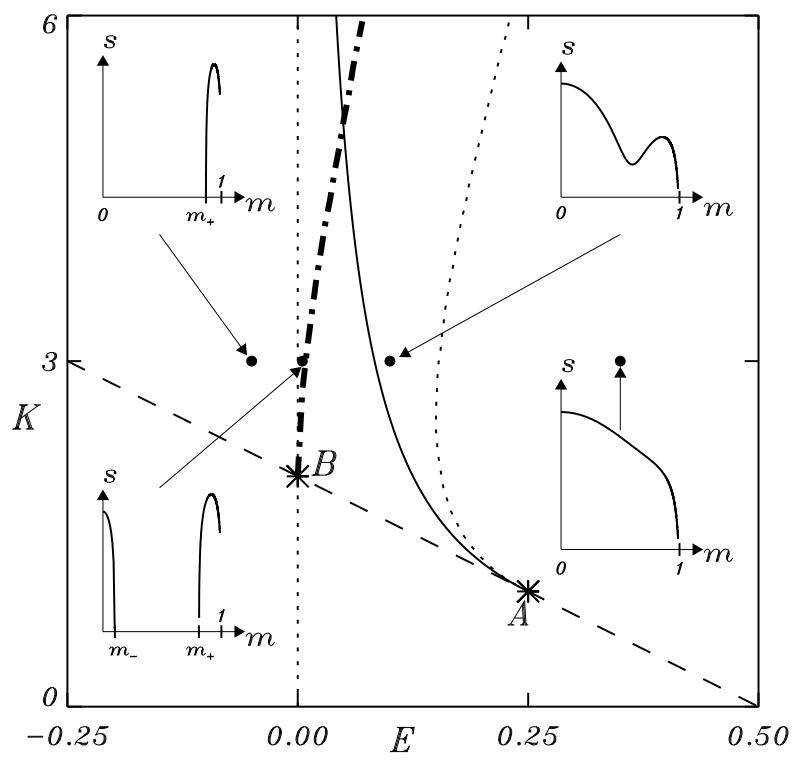

FIG. 4: Phase diagram of the mean field model (44). The dash-dotted curve corresponds to the first order phase transition line, issued from the point $B(0,2)$. As in Fig. 2, the solid curve indicates the right border of the region $R 3$, where the phase space is disconnected. The dashed line corresponds to the $K=2-4 E$. The dotted line issued from the point $A(1 / 4,1)$ represents the metastability line for the magnetized state, while the $E=0$ vertical dotted line is also the metastability line for the non-magnetic state. The four insets represent the entropy $s$ versus the magnetization $m$ for the four energies: $E=-0.05,0.005,0.1$ and 0.35 , when $K=3$.

Figure 4 also shows the metastability line (the dotted line starting at point $A(1 / 4,1)$ ), for the magnetized state $m_{e q} \neq 0$. To the right of this metastability line, there is no metastable state (local entropy maximum for any $m>0$, see bottom-right inset in Fig. (4) while a metastable state (local maximum) exists at some non vanishing magnetization on the other side (see top-right inset in Fig. (4). Finally, the vertical dotted line $E=0$ corresponds to the metastability line of the non-magnetic state $m=0$.

One of the key issues we would like to address is the possible links between the breakdown of phase space connectivity and thus ergodicity breaking, on the one hand, and the phase transition, on the other hand. Obvious general properties do exist: a region of parameters where the phase space is disconnected corresponds to a region where metastable states do exist. Let us justify this statement. At the boundary of any connected domain, when the order parameter is close to its boundary value $m_{b}$, there is a single accessible state. In a model with continuous variables, like the one we study in this paper, this leads to a divergence of the entropy. In this case the singularity of the entropy is proportional to $\ln \left(m-m_{b}\right)$

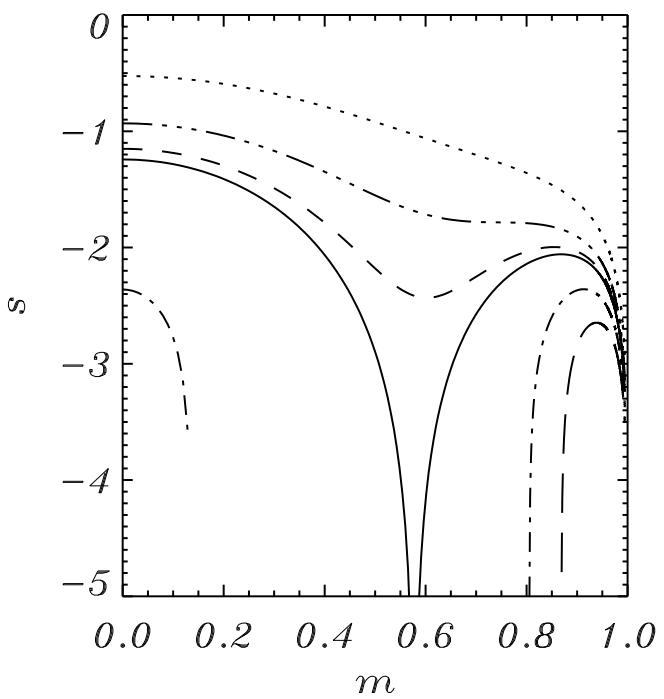

FIG. 5: Entropy $s$ versus magnetization $m$ for $K=3$ and several energy values. The different curves correspond, from top to bottom, to $E=0.35$ (dotted), 0.155 (dash-triple dotted, metastability limit for the magnetized state), 0.1 (dashed), $E=1 /(4 K)=1 / 12$ (solid, appearence of the gap), 0.0089 (dash-dotted, first order phase transition), -0.05 (long dashed). This picture demonstrates that gaps in the accessible states develop as the energy is lowered.

(see for instance equation (9)). For a model with discrete variables, like an Ising model, the entropy would no more reach $-\infty$ as $m$ tends to $m_{b}$, but would rather take a finite value. However, the singularity would still exist and would then be proportional to $\left(m-m_{b}\right) \ln \left(m-m_{b}\right)$. In both cases, of discrete and continuous variables, at the boundary of any connected domain, the derivative of the entropy as a function of the order parameter tends generally to $\pm \infty$. As a consequence, entropy extrema cannot be located at the boundary. Thus, a local entropy maximum (metastable or stable) does exist in a region of parameters where the phase space is disconnected.

Hence, there is an entropy maximum (either local or global) in any connected domain of the phase space. For instance, considering the present model, in Fig. 4 the area $R 3$ is included in the area where metastable states exist (bounded by the two dotted lines and the dashed line). In such areas where metastable states exist, one generically expects first order phase transitions. Thus the breakdown of phase space connectivity will be generically associated to first order phase transition, as exemplified by the present study. However, this is not necessary, one may observe metastable states without first order phase transitions, or first order phase transitions without connectivity breaking.

A very interesting question is related to the critical points $A$ and $B$ shown in Fig. 4. As observed in the phase diagram, the end point for the line of first order 
phase transition (point $B$ ) corresponds also to a point where the boundary of the region where the phase space is disconnected is not smooth. Similarly, the end point for the line of appearance of metastable states (point $A$ ) is also a singular point for the boundary of the area where the phase space is disconnected. It is thus possible to propose the conjecture that such a relation is generic, and that it should be observed in other systems where both first order phase transitions and phase space ergodicity breaking do occur.

\section{DYNAMICS}

The feature of disconnected accessible magnetization intervals, which is typical of systems with long-range interactions, has profound implications on the dynamics. In particular, starting from an initial condition which lies within one of these intervals, local dynamics is unable to move the system to a different accessible interval. A macrostate change would be necessary to carry the system from one interval to the other. Thus the ergodicity is broken in the microcanonical dynamics even at finite $N$.

In Ref. 4] this point has been demonstrated using the microcanonical Monte-Carlo dynamics suggested by Creutz [8]. Here, we use the Hamiltonian dynamics given by the equation of motions

$$
\begin{aligned}
\dot{p}_{n} & =-\frac{\partial H}{\partial \theta_{n}}=-N\left(m \frac{\partial m}{\partial \theta_{n}}-K m^{3} \frac{\partial m}{\partial \theta_{n}}\right) \\
& =N\left(1-K m^{2}\right)\left(\sin \theta_{n} m_{x}-\cos \theta_{n} m_{y}\right) .
\end{aligned}
$$

We display in Fig. 6 the evolution of the magnetization for two cases, since we have shown above that the gap opens up when $E$ decreases. The first case corresponds to the domain $R 1$, in which the accessible magnetization domain is the full interval $[0,1]$. Fig. 6a presents the time evolution of the modulus $m$ of the order parameter (2) versus time. The magnetization switches between the metastable state $m=0$ and the stable one $m_{e q}>0$. This is possible because the number of particles is small $(N=20)$ and, as a consequence, the entropy barrier (see the inset) can be overcome. Considering a system with a small number of particles allows to observe flips between local maxima, while such flips would be less frequent for larger $N$ values.

In the other case, we consider a stable $m=0$ state which is disconnected from the metastable one. It makes the system unable to switch from one state to the other. Note that this feature is characteristic of the microcanonical dynamics, since an algorithm reproducing the canonical dynamics would allow the crossing of the forbidden region (by moving to higher energy states, which is impossible in the microcanonical ensemble). The result of two different numerical simulations is reported in Fig. 6b. One is initialized with a magnetization within $\left[0, m_{-}\right]$, while the other corresponds to an initial magnetization

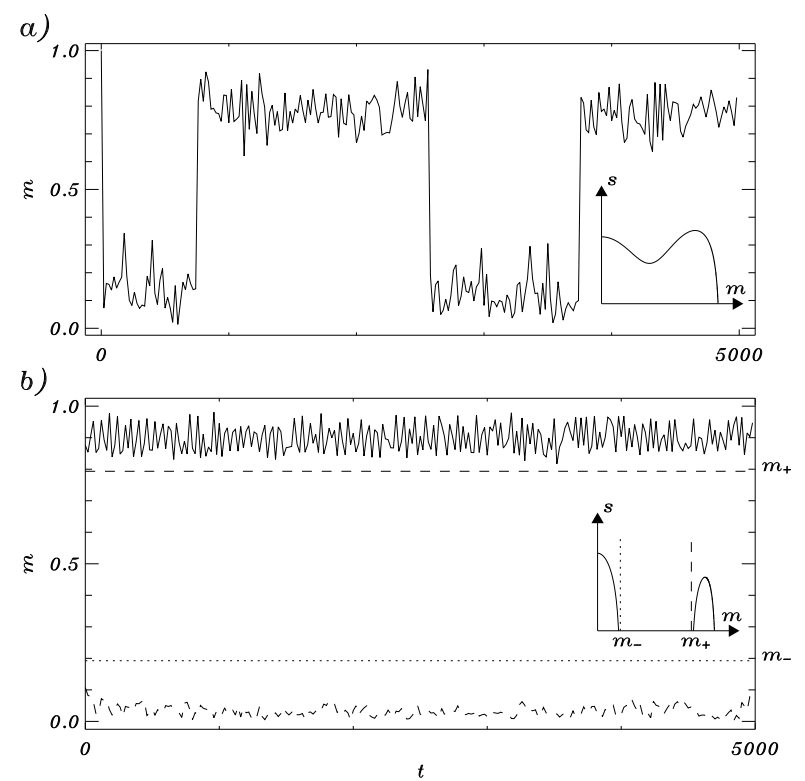

FIG. 6: Time evolution of the magnetization $m$ (the entropy of the corresponding cases is plotted as an inset). Panel a) corresponds to the case $E=0.1$ and $K=8$, while panel b) to $E=0.0177$ and $K=3$. In panel b), two different initial conditions are plotted simultaneously: the solid line corresponds to $m(t=0)=0.1$ while the dashed line to $m(t=0)=0.98$. The dashed (resp. dotted) line in panel b) corresponds to the line $m=m_{+} \simeq 0.794$ (resp. $m=m_{-} \simeq 0.192$ ).

close to $m=1$ (i.e. within $\left[m_{+}, 1\right]$ ). One clearly sees that the dynamics is blocked in one of the two possible regions, and not a single jump is visible over a long time span. This is a clear evidence of ergodicity breaking.

\section{CONCLUSIONS}

We have found that for sufficiently low energy, gaps open up in the magnetization interval, to which no microscopic configurations corresponds. Thus the phase space breaks into disconnected regions. Within the microcanonical dynamics the system is trapped in one of these regions, leading to a breakdown of ergodicity even in finite systems.

Ergodicity breaking implies that an ensemble average will not be well reproduced by a time average, usually simpler to get from the experimental or numerical point of view. Such a discrepancy, forbidden a priori for shortrange interacting system, might be a typical case for systems with long-range interactions.

Of high interest would be of course to study systems with both short and long-range interactions since they usually compete in standard condensed matter systems 9]. Such a study could suggest an experimental system where ergodicity breaking might be observed. 


\section{Acknowledgments}

This work was supported by the ANR program STATFLOW (ANR-06-JCJC-0037-01) and by the Minerva Foundation with funding from the Federal Ministry for Education and Research. Visit of F. B. to the Weizmann
Institute has been supported by the Albert Einstein Minerva Center for Theoretical Physics. This work is also funded from the PRIN05 grant of MUR-Italy Dynamics and thermodynamics of systems with long-range interactions.
[1] T. Dauxois, S. Ruffo, E. Arimondo, M. Wilkens (Eds), Dynamics and Thermodynamics in systems with longrange interactions, Lecture Notes in Physics 602, Springer (2002).

[2] F. Borgonovi, G. L. Celardo, M. Maianti, E. Pedersoli, J. Stat. Phys. 116, 1435 (2004).

[3] F. Borgonovi, G.L. Celardo, A. Musesti, R. TrasartiBattistoni and P. Vachal, Phys. Rev. E, 73, 026116 (2006).

[4] D. Mukamel, S. Ruffo, N. Schreiber, Phys. Rev. Lett. 95, 240604 (2005).
[5] P. de Buyl, D. Mukamel, S. Ruffo, AIP Conf. Proceedings, 800, 533 (2005).

[6] J. Barré, F. Bouchet, T. Dauxois, S. Ruffo, Journal of Statistical Physics 119, 677-713 (2005).

[7] G. L. Celardo, J. Barré, F. Borgonovi and S. Ruffo, Phys. Rev. E, 73, 011108 (2005)

[8] M. Creutz, Phys. Rev. Lett. 50, 1411 (1983).

[9] A. Campa, R. Khomeriki, D. Mukamel and S. Ruffo, Phys. Rev. B, 76, 064415 (2007). 